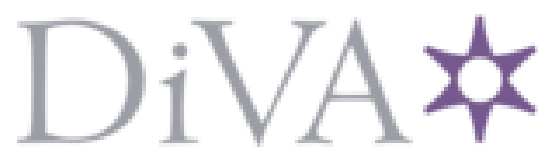

http://www.diva-portal.org

This is the published version of a paper published in Physical Review E. Statistical, Nonlinear, and Soft Matter Physics.

Citation for the original published paper (version of record):

Tian, C., Zhang, L. (2013)

Delay-driven irregular spatiotemporal patterns in a plankton system.

Physical Review E. Statistical, Nonlinear, and Soft Matter Physics, 88(1): 012713

http://dx.doi.org/10.1103/PhysRevE.88.012713

Access to the published version may require subscription.

N.B. When citing this work, cite the original published paper.

Permanent link to this version:

http://urn.kb.se/resolve?urn=urn:nbn:se:umu:diva-79424 


\title{
Delay-driven irregular spatiotemporal patterns in a plankton system
}

\author{
Canrong Tian* \\ Department of Basic Sciences, Yancheng Institute of Technology, Yancheng 224003, China \\ Lai Zhang ${ }^{\dagger}$ \\ Department of Mathematics and Mathematical Statistics, Umeå University, SE-90187 Umeå, Sweden \\ (Received 2 February 2013; revised manuscript received 6 June 2013; published 11 July 2013)
}

\begin{abstract}
An inhomogeneous distribution of species density over physical space is a widely observed scenario in plankton systems. Understanding the mechanisms resulting in these spatial patterns is a central topic in plankton ecology. In this paper we explore the impact of time delay on spatiotemporal patterns in a prey-predator plankton system. We find that time delay can trigger the emergence of irregular spatial patterns via a Hopf bifurcation. Moreover, a phase transition from a regular spiral pattern to an irregular one was observed and the latter gradually replaced the former and persisted indefinitely. The characteristic length of the emergent spatial pattern is consistent with the scale of plankton patterns observed in field studies.
\end{abstract}

DOI: 10.1103/PhysRevE.88.012713 PACS number(s): 87.23.-n, 05.45.-a, 82.39.-k, 89.75.-k

\section{INTRODUCTION}

A heterogeneous distribution of species abundance across different spatial scales is a pronounced scenario in natural ecosystems. Understanding the mechanisms responsible for such pattern formation is one of the central problems in ecology. During recent decades, pattern formation has been a major interest of theorists and experimentalists, and it is generally thought that spatial patterns form as a consequence of the interplay of various physical and biological processes [1]. It has been shown that ecological systems even in perfectly homogeneous surroundings can exhibit inhomogeneous patterns [2-4]. Due to widespread environmental noise [5-10], no ecological system is really homogeneous. Thus, exploring what contributes to the emergence of self-organized spatial patterns undoubtedly aids the understanding of pattern formation.

Spatial patterns were first investigated by Turing [11] in a reaction-diffusion chemical system. The idea is that an initially uniform distribution of reacting components could become unstable as a result of diffusion, whereby a stationary spatial pattern arises. This is known as diffusion-driven instability or Turing instability, and this idea has quickly spread to ecological systems [12]. Similar ideas were used to explain the ecological spatial patterns arising, for example, in plankton systems [13] and in semiarid vegetation systems [14].

Apart from the Turing mechanism, attention to spatial pattern formation has also shifted to non-Turing mechanisms, e.g., the Hopf bifurcation [15]. Particular attention has been given to mechanisms that result in irregular spatiotemporal patterns (e.g., $[16,17])$. Irregular spatial patterns are considered ecologically relevant and are well consistent with natural spatial irregularities of species abundance distributions [18]. Thus, an understanding of which mechanisms may result in the emergence of irregular spatiotemporal patterns is of ecological importance. Baurmann et al. [15] showed that irregular patterns arise in the neighborhood of the Turing-Hopf

\footnotetext{
*Corresponding author: tiancanrong@163.com

†lai.zhang@math.umu.se
}

bifurcation, but Banerjee and Petrovskii [18] found in a ratio-dependent predator-prey model that the Hopf bifurcation is essential for the onset of irregular spatiotemporal patterns.

Theoretical studies of spatiotemporal patterns are usually concentrated on ecological interactions that occur spontaneously without any delay. Growing evidence suggests that delay feedback is an important factor affecting the dynamics of ecological systems as the conversion from food intake to reproduction is not instantaneous, but mediated by some time lag required for the growth and maturation of individuals. Time delay was first considered in a prey-predator system by Volterra [19], who showed that under certain conditions a spatial distribution may possess oscillatory behavior. Since then, several theoretical investigations have demonstrated the emergence of delay-driven spatial patterns (e.g., [20-29]). An experimental study of delay feedback in [30] found that time delay could induce a spiral wave, and when the delay between the registration of a wave front at the measuring point and triggering of the stimulus was increased, so was the diameter of the synchronized trajectory of the spirals.

Motivated by the experimental observation of spiral waves, we explore the effect of time delay on irregular spatiotemporal patterns in a planktonic prey-predator system. Our analysis mainly reveals that, with a proper time delay, regular spiral patterns emerge for both populations in the beginning, but they gradually break down, and consequently irregular patterns appear. The regular and irregular patterns can coexist for a sufficiently long period before the irregular patterns ultimately replace the regular ones and persist forever. We argue that the emerging spatial pattern is consistent with the scale of spatial plankton abundance patterns observed in field studies.

\section{THE PLANKTON MODEL}

Phytoplankton and zooplankton are floating organisms living in the sea, in freshwater lakes, and in larger rivers [31,32]; they form the basis for the food webs in the ocean. The population dynamics of plankton species was traditionally described using Lotka-Volterra equations (e.g., [13]). Taking into account the results of field and laboratory observations 
on plankton systems [33], it is more realistic to assume that the phytoplankton species obey logistic growth in the absence of zooplankton and that zooplankton consume phytoplankton with a Holling type-II functional response $[1,34]$. In this case the reaction-diffusion plankton model can be formulated as follows:

$$
\begin{aligned}
& \frac{\partial u}{\partial t}=D \Delta u+\frac{\alpha}{b} u(b-u)-\gamma \frac{u}{u+H} v, \\
& \frac{\partial v}{\partial t}=D \Delta v+\beta \gamma \frac{u}{u+H} v-\mu v,
\end{aligned}
$$

where $u(x, t)$ and $v(x, t)$ are, respectively, the densities of prey and predators at space coordinate $x$ and time $t . \alpha$ and $b$ are the intrinsic growth rate and carrying capacity of the prey. $\gamma$ indicates the capture rate of the predator and $H$ is the halfsaturation abundance of prey. $\beta$ denotes the efficiency of food utilization and $\mu$ is the background mortality of the predator. $D$ is the diffusion coefficient for both prey and predators. All parameters are positive constants.

To minimize the number of parameters involved in the model, we introduce dimensionless variables by setting

$$
\tilde{u}=u / b, \tilde{v}=v \gamma /(\alpha b), \tilde{t}=\alpha t, \tilde{x}=\sqrt{\alpha / D} x .
$$

Omitting the tildes, we arrive at a dimensionless plankton system:

$$
\begin{aligned}
& \frac{\partial u}{\partial t}=\Delta u+u(1-u)-\frac{u}{u+h} v \\
& \frac{\partial v}{\partial t}=\Delta v+\kappa \frac{u}{u+h} v-m v
\end{aligned}
$$

where $h=H / b, m=\mu / \alpha$, and $\kappa=\beta \gamma / \alpha$.

The above model has received much attention, and many interesting results derived from it have been reported. For a detailed description of the model and of results pertaining to it we refer readers to the review paper by Medvinsky et al. [1]. A limitation of this model is that it assumes that future abundances of species depend only on their present states. Recently, a number of authors have found in predator-prey systems that a population's past history has a residual effect on the course of future species abundances [35-39]. In fact, the conversion from food energy to reproduction requires some time. Taking such effects into consideration, we refine system (3) by adding a time delay $\tau$ :

$$
\begin{aligned}
& \frac{\partial u}{\partial t}=\Delta u+u(1-u)-\frac{u}{u+h} v:=f(u, v), \\
& \frac{\partial v}{\partial t}=\Delta v+\kappa \frac{u_{\tau}}{u_{\tau}+h} v-m v:=g\left(u_{\tau}, v\right),
\end{aligned}
$$

where $(u)_{\tau} \equiv u(x, t-\tau)$, and $\tau$ is a positive constant. In the following sections, system (4) is analyzed under zero-flux boundary conditions, which biologically means that there is no population flux across the boundary.

In a plankton system, spatial patterns can be formed due to many factors. It is generally thought that the spatial dynamics is shaped by the interplay of physical factors such as light, temperature, and hydrodynamics, and biological factors such as nutrient uptake and predation by fish [1]. Since our interest is in the biological interactions we exclude physical factors by assuming homogeneous physical surroundings. Petrovskii and Malchow [34] and Medvinsky et al. [1] both demonstrated that irregular spatiotemporal patterns can be triggered solely by nonlinear prey-predator interactions. It remains unclear if such complex spatial dynamics can also be excited by time delay. Thus in the following sections we focus on the parts of parameter space where no spatiotemporal patterns can arise if time delay is absent.

\section{DELAY-DRIVEN SPATIOTEMPORAL PATTERNS}

In this section we derive the necessary conditions for the emergence of spatiotemporal patterns by means of linear stability analysis. Setting $\delta u=u-u^{*}$ and $\delta v=v-v^{*}$, where $\left(u^{*}, v^{*}\right)$ is the uniform equilibrium of system (4), and linearizing around it in $u$ and $v$ gives

$$
\begin{aligned}
& \frac{\partial \delta u}{\partial t}=\Delta \delta u+f_{u} \delta u+f_{v} \delta v, \\
& \frac{\partial \delta v}{\partial t}=\Delta \delta v+g_{u_{\tau}} \delta u+f_{v} \delta v,
\end{aligned}
$$

where $f_{u}=\left.\frac{\partial f}{\partial u}\right|_{\left(u^{*}, v^{*}\right)}, \quad f_{v}=\left.\frac{\partial f}{\partial v}\right|_{\left(u^{*}, v^{*}\right)}, \quad g_{u_{\tau}}=\left.\frac{\partial g}{\partial u_{\tau}}\right|_{\left(u^{*}, v^{*}\right)}$, and $g_{v}=\left.\frac{\partial g}{\partial v}\right|_{\left(u^{*}, v^{*}\right)}$.

Since we have zero-flux boundary conditions, the eigenfunction of (5) is

$$
(\delta u, \delta v)=\left(\delta u^{*}, \delta v^{*}\right) e^{\lambda t} \cos \mathbf{k} x,
$$

where $\lambda$ is the frequency and $\mathbf{k}$ is the wave number in the $x$ direction. Substituting this form into (5), we obtain the following matrix equation for the eigenvalues:

$$
\left(\begin{array}{cc}
\lambda-f_{u}+k^{2} & -f_{v} \\
-g_{u_{\tau}} e^{-\lambda \tau} & \lambda-g_{v}+k^{2}
\end{array}\right)\left(\begin{array}{l}
\delta u^{*} \\
\delta v^{*}
\end{array}\right)=\left(\begin{array}{l}
0 \\
0
\end{array}\right) .
$$

Therefore, we have the following characteristic equation for the eigenvalues:

$$
\Delta(\lambda, \tau):=\lambda^{2}+A \lambda+B+C e^{-\lambda \tau}=0,
$$

where $A=-f_{u}-g_{v}+2 k^{2}, \quad B=\left(-f_{u}+k^{2}\right)\left(-g_{v}+k^{2}\right)$, $C=-f_{v} g_{u_{\tau}}$, and $k^{2}=\mathbf{k} \cdot \mathbf{k}$.

Note that in the case of $\tau=0$, i.e., when the time delay is absent, the characteristic equation is reduced to the following quadratic equation:

$$
\lambda^{2}+A \lambda+B+C=0 .
$$

Since our aim is to understand the role of time delay in the formation of spatiotemporal chaos, we assume that the following conditions hold:

$$
A<0 \text { and } B+C>0,
$$

under which the equilibrium is stable for system (3).

We now consider the effect of time delay and derive the conditions under which the uniform equilibrium of system (4) undergoes Hopf bifurcation. According to the instability theory of [40], in order to prove the instability of the uniform equilibrium, we need only to show that there exist two roots of (7) such that $\Delta(i \omega, \tau)=0$, where $i \omega$ is purely imaginary and $\tau$ is positive and real.

If $i \omega$ is a root of (7), then we have

$$
\begin{aligned}
& \omega^{2}-B=C \cos \omega \tau, \\
& A \omega=C \sin \omega \tau,
\end{aligned}
$$


which leads to

$$
\omega^{4}+\left(A^{2}-2 B\right) \omega^{2}+B^{2}-C^{2}=0 .
$$

Noticing that Eq. (11) is a quadratic equation in $\omega^{2}$ and that by the Descartes rule of signs, Eq. (11) has a unique positive solution $\omega_{c}$ if and only if

$$
B<C,
$$

we can compute the solution of (11) under these conditions to be

$$
\omega_{c}=\frac{\left\{2 B-A^{2}+\left[\left(A^{2}-2 B\right)^{2}-4\left(B^{2}-C^{2}\right)\right]^{1 / 2}\right\}^{1 / 2}}{\sqrt{2}} .
$$

Therefore, $\Delta(i \omega, \tau)=0$ has a pair of roots of $\omega_{c}$ and $\tau_{c}$, where

$$
\tau_{c}=\frac{\arccos \left[\left(\omega_{c}^{2}-B\right) / C\right]}{\omega_{c}} .
$$

In view of the instability theory of [40], to ensure that the system (4) is unstable, the lower bound of $\tau$ must satisfy

$$
\tau>\tau_{c} .
$$

Moreover, it is easy to verify that the uniform equilibrium $\left(u^{*}, v^{*}\right)$ of system (4) undergoes a Hopf bifurcation at the threshold $\tau_{c}$.

In conclusion, when the time delay is greater than the critical value $\tau_{c}$ spatiotemporal instability in the two-component reaction-diffusion system occurs. In the coming section, we shall demonstrate numerically that the dynamics of (4) exhibits a spatiotemporal pattern.

\section{IRREGULAR PATTERN FORMATION}

\section{A. Parameter space of irregular patterns}

We now explore the parameter space to find the subspace where the fulfillment of conditions (9) and (12) is guaranteed. Considering the specific plankton model (4), we have

$$
\begin{aligned}
& f_{u}=u^{*}\left(-1+\frac{1-u^{*}}{u^{*}+h}\right), \quad f_{v}=-\frac{u^{*}}{u^{*}+h}, \\
& g_{u}=g_{u_{\tau}}=\kappa h \frac{1-u^{*}}{u^{*}+h}, \quad g_{v}=0 .
\end{aligned}
$$

Direct computation shows that the two conditions (9) and (12) can be fulfilled if the parameters satisfy the following relation:

$$
\frac{\kappa-m}{\kappa+m}<h<\frac{\kappa-m}{m} .
$$

In this paper, we will use the following parameter values:

$$
\kappa=2, \quad m=0.8, h=0.5 \text {. }
$$

These parameter values are refined slightly from [34] such that conditions (9) and (12) are satisfied. Therefore if system (4) exhibits any spatiotemporal chaos it must be due to the time delay.

For this particular choice, the positive uniform equilibrium is given by

$$
\left(u^{*}, v^{*}\right)=(1 / 3,5 / 9) \approx(0.3333,0.5556),
$$

and the critical time delay is $\tau_{c}=0.1177$. Therefore the equilibrium of the system (4) is stable (unstable) when $\tau<$ $\tau_{c}=0.1177$ ( $\left.\tau \geqslant 0.1177\right)$. In addition we can see from Fig. 1 that the critical time delay for a Hopf bifurcation increases

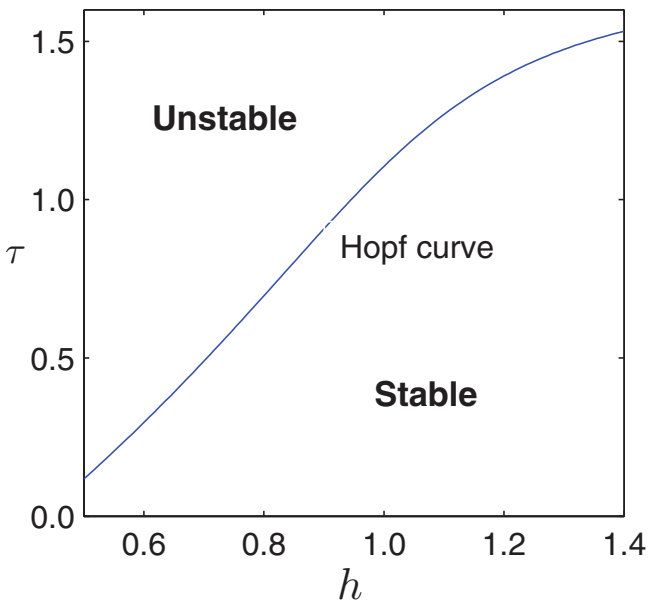

FIG. 1. (Color online) Bifurcation diagram in the $\tau$ - $h$ parameter space. The solid curve indicates the critical bifurcation point $\tau_{c}$ as a function of $h$.

with increasing $h$. We set $\tau=0.3$ in the remainder of this paper.

\section{B. Numerical simulations}

Using the Euler forward finite difference method, we give our numerical results based on the formulas in $[41,42]$. We consider system (4) in a fixed spatial domain $\left[0, L_{x}\right] \times\left[0, L_{y}\right]$ $\left(L_{x}=900, L_{y}=300\right)$ and solve it on a grid with $900 \times 300$ points. The space step is $\Delta x=\Delta y=1$, and the time step is $\Delta t=0.1$. At the beginning of the simulation we assume that the two species are spread over the whole domain. It is well known that the spatial dynamics of system (4) is affected by an initial spatial perturbation. If the initial distribution is spatially homogeneous, then the homogeneous distribution of species density will remain forever, and therefore it is not so interesting.

Our numerical experiments start with an inhomogeneous distribution. To this end, we take the following initial distribution:

$$
\begin{aligned}
& u(x, y, t)=u^{*}-\epsilon_{1}(x-0.1 y-225)(x-0.1 y-675), \\
& v(x, y, t)=v^{*}-\epsilon_{2}(x-445)-\epsilon_{3}(y-150),
\end{aligned}
$$

where $\epsilon_{1}=2 \times 10^{-7}, \epsilon_{2}=3 \times 10^{-5}$, and $\epsilon_{3}=1.2 \times 10^{-4}$. Snapshots of the spatial abundance distribution of the predator species are shown in Fig. 2 (spatial patterns of the prey abundance are qualitatively similar except for the early stages of the process when the influence of the initial condition dominates). We see a gradual formation of an irregular spatial pattern starting as regular spiral patterns. As the initial condition contains two critical points $(245.122,201.22),(684.146,91.463)$, that satisfy $\left.u\left(x_{c}, y_{c}\right)\right|_{t=0}=u^{*},\left.v\left(x_{c}, y_{c}\right)\right|_{t=0}=v^{*}$, in the initial structure [Fig. 2(a)], two spirals emerge around the critical points [Fig. 2(b)]. The spirals grow slightly in the beginning, before disappearing [Fig. 2(c)]. The disappearance of the structures takes place around the center of the spirals where "embryos" [Fig. 2(d)], once they appear, grow steadily [Fig. 2(e)]. Eventually, the irregular spatial pattern prevails over the whole domain [Fig. 2(f)]. The dynamic process of Fig. 2 is described in the Supplemental Material [43]. 
$\begin{array}{llllll}0.53 & 0.54 & 0.55 & 0.56 & 0.57 & 0.58\end{array}$

(a)

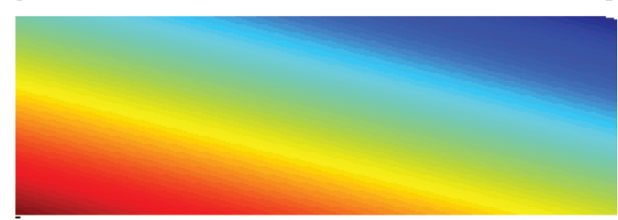

(b)

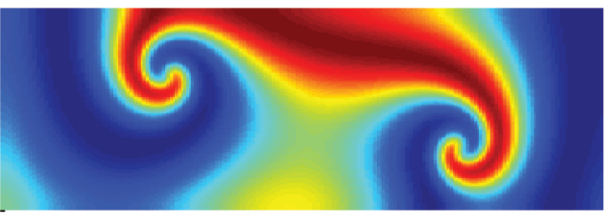

(c)

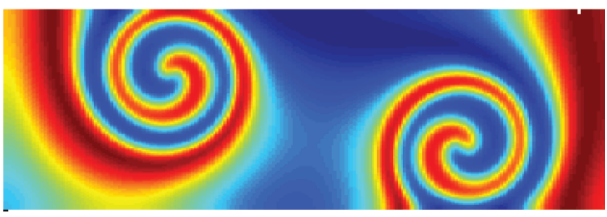

(d)

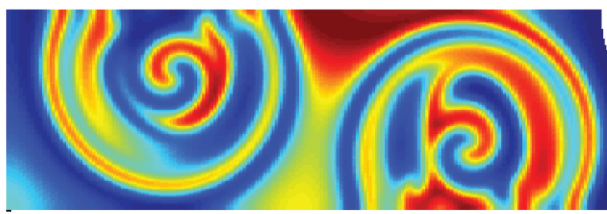

(e)

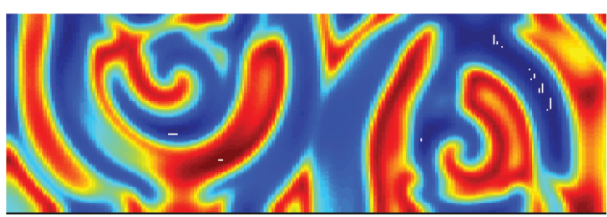

(f)

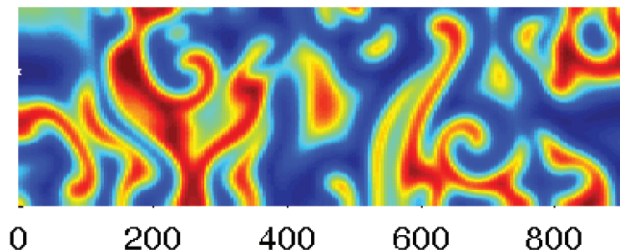

FIG. 2. (Color online) Spatial distribution of predators for (a) $t=0$, (b) $t=500$, (c) $t=1000$, (d) $t=1500$, (e) $t=2000$, and (f) $t=2500$. The parameters are given in the text. From (a) to (c), the distribution evolves to the spiral. From (d) to (f), an irregular patchy structure arises as a result of the destruction of the spiral.

Now we explore this regime of the system dynamics in more detail. This can be seen from Figs. 3 and 4. Figure 3 demonstrates the spatially averaged population density over time, which is thought to be an appropriate indicator of irregular spatial dynamics [34]. It clearly shows that after a sufficiently long time, the dynamics of the spatial pattern is irregular. Figure 4 shows the moving trajectory in the phase plane obtained at a fixed point $(x, y)=(450,150)$. Note that the phase planes are qualitatively similar at other fixed points. The local phase plane shows that the trajectory fills nearly the whole domain inside the limit cycle. Inside the limit cycle irregular spatiotemporal oscillations occur, while in the neighborhood of the limit cycle we see instead regular spatiotemporal oscillations with roughly constant amplitude. Until the irregular spatial pattern spreads over the whole domain, there exists a distinct boundary that divides the

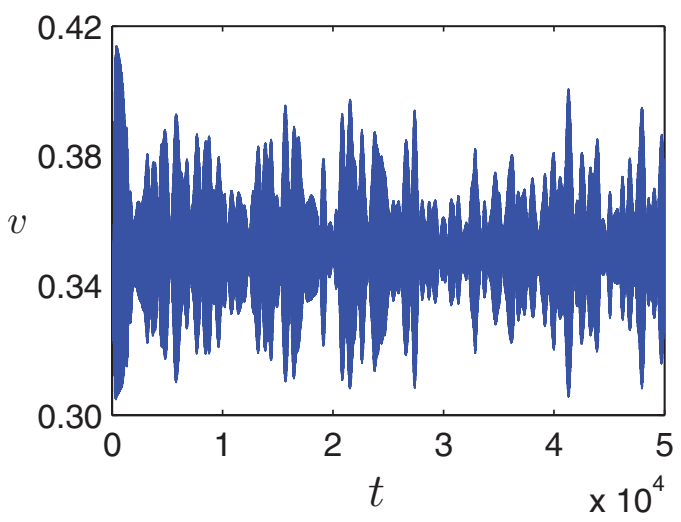

FIG. 3. (Color online) Time series of spatially averaged predator density.

domain into two regions with different dynamic regimes, namely, the "jagged" and smooth patterns (see also Ref. [34]). Later the boundary moves steadily at roughly constant speed and the jagged irregular region eventually occupies the whole region. However, before the irregular region replaces the regular one, the two dynamic regimes can coexist for a long period of time. The displacement of the regular regime by the irregular one demonstrates a phase transition between regular and irregular phases.

Combining the observations from Figs. 2, 3 and 4, we see that a time delay can eventually trigger the emergence of an irregular spatiotemporal pattern, and once it appears, it persists forever. We now determine how the theoretical finding agrees with the results from field observations. Clearly, Fig. 2(f) shows that the characteristic length of the spatial pattern is roughly between 20 and 40 in dimensionless units. Equation (2) indicates that the magnitude of these values in dimensional units is dependent on the maximal phytoplankton growth rate $\alpha$ and the diffusive coefficient $D$. The value of $\alpha$ ranges from $10^{-5}$ to $4 \times 10^{-5} \mathrm{~s}^{-1}$, at its peak [1]. The turbulent diffusion $D$, although dependent on the scale of the phenomenon for the open sea [44-46], varies much less in coastal regions such as bights and harbors, and in these regions we have

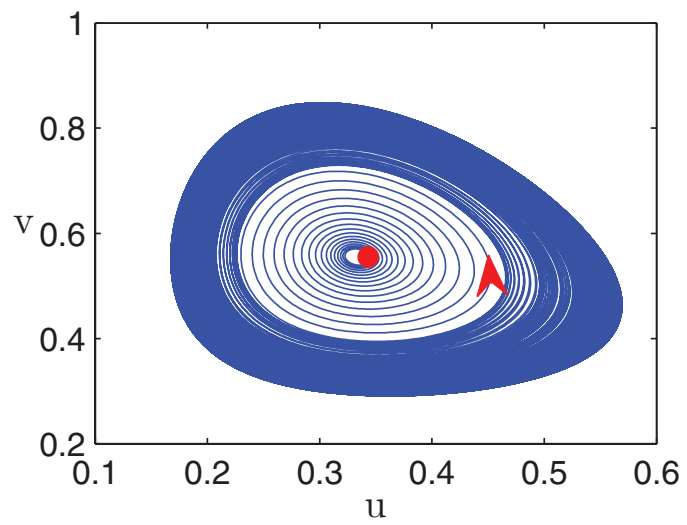

FIG. 4. (Color online) Phase plane of the system (4) at a fixed spatial location $(450,150)$ inside the domain occupied by irregular spatiotemporal oscillations. The arrow indicates the direction of movement of the trajectory and the solid circle shows the starting point. 
$D=10^{3} \mathrm{~cm}^{2} \mathrm{~s}^{-1}[44,47]$. Substituting these estimates for $D$ and $\alpha$ into Eq. (2), we find that the dimensionless unit length corresponds to approximately $50-100 \mathrm{~m}$ in the original dimensional units, which gives a characteristic length of the spatial patterns of the order of $1 \mathrm{~km}$. This scale of the spatial patterns agrees well with field observations $[33,48,49]$.

\section{DISCUSSION}

Irregular spatiotemporal patterns have important implications for population dynamics $[16,17]$, and are widely thought to be of high ecological relevance as they can emerge under less restrictive conditions than Turing patterns [18]. Since most ecosystems, if not all, are affected by environmental noise [5-10], the emergence of irregular spatial patterns might be very likely to occur in real ecological communities.

In our prey-predator plankton system, we have shown that irregular spatial patterns can be excited near the Hopf bifurcation point of a time delay, beyond which the spatially uniform equilibrium loses its stability. This finding confirms once again that a Hopf bifurcation is a necessary condition for the onset of irregular spatiotemporal patterns [18]. The reason for the appearance of the irregular patterns that result from the destruction of a regular spiral pattern remains poorly understood, but a plausible explanation might be that a dynamical system with delay is many dimensional, and therefore a few modes selected by the boundary conditions actively interact, leading to the breakup of the coherent structure of the spirals [50].

The emergent irregular patterns are fairly robust. Use of periodic boundary conditions gives rise to similar patterns. In addition, the domain size and initial conditions are not crucial for exciting irregular patterns, but the number of spirals is positively correlated with the number of critical points appearing in the initial perturbations. Since the predicted irregular pattern shows good agreement with field observations, the proposed approach may have broader applicability to other reaction-diffusion systems with time delay, such as those with competitive and mutualistic interactions.

\section{ACKNOWLEDGMENTS}

C.T. acknowledges financial support by the PRC Grant No. NSFC 11201406, and the Qinglan Project. L.Z. gratefully acknowledges financial support from the Swedish Kempe Foundations. We also thank Uffe Høgsbro Thygesen and three reviewers for their valuable comments.
[1] A. B. Medvinsky, S. V. Petrovskii, I. A. Tikhonova, H. Malchow, and B. L. Li, SIAM Rev. 44, 311 (2002).

[2] H. Malchow, Proc. R. Soc. London, Ser. B 251, 103 (1993).

[3] P. K. Maini, K. J. Painter, and H. N. P. Chau, J. Chem. Soc., Faraday Trans. 93, 3601 (1997).

[4] H. Malchowa, B. Radtkea, M. Kallachea, Alexander B. Medvinskyb, Dmitry A. Tikhonovb, and Sergei V. Petrovskiic, Nonlin. Anal. RWA 1, 53 (2000).

[5] B. Spagnolo, D. Valenti, and A. Fiasconaro, Math. Biosci. Eng. 1, 185 (2004).

[6] D. Valenti, L. Schimansky-Geier, X. Sailer, and B. Spagnolo, Eur. Phys. J. B 50, 199 (2006).

[7] D. Valenti, A. Fiasconaro, and B. Spagnolo, Acta Phys. Pol. B 35, 1481 (2004).

[8] B. Spagnolo, A. Fiasconaro, and D. Valenti, Fluct. Noise Lett. 3, L177 (2003).

[9] D. Valenti, G. Denaro, A. La Cognata, B. Spagnolo, A. Bonanno, G. Basilone, S. Mazzola, S. Zgozi, and S. Aronica, Acta Phys. Pol. B 43, 1227 (2012).

[10] G. Denaro, A. La Cognata, D. Valenti, B. Spagnolo, A. Bonanno, W. Basilone, S. Mazzola, S. Zgozi, and S. Aronica, Ecol. Complex. 13, 21 (2013).

[11] A. Turing, Philos. Trans. R. Soc., B 237, 37 (1952).

[12] L. A. Segel and J. L. Jackson, J. Theor. Biol. 37, 545 (1972).

[13] S. A. Levin and L. A. Segel, Nature (London) 259, 659 (1976).

[14] C. A. Klausmeier, Science 284, 1826 (1999).

[15] M. Baurmann, T. Gross, and U. Feudel, J. Theor. Biol. 245, 220 (2007).

[16] M. Pascual, Proc. R. Soc. London, Ser. B 251, 17 (1993).

[17] J. A. Sherratt, Ecol. Lett. 4, 30 (2001).

[18] M. Banerjee and S. Petrovskii, Theor. Ecol. 4, 37 (2011).
[19] V. Volterra, Lecons sur la Théorie Mathématique de la Lutte par la Vie (Gauthier-Villars, Paris, 1931).

[20] S. Sen, P. Ghosh, S. S. Riaz, and D. S. Ray, Phys. Rev. E 80, 046212 (2009).

[21] S. Boccaletti, D. Maza, H. Mancini, R. Genesio, and F. T. Arecchi, Phys. Rev. Lett. 79, 5246 (1997).

[22] C. Beta, M. Bertram, A. S. Mikhailov, H. H. Rotermund, and G. Ertl, Phys. Rev. E 67, 046224 (2003).

[23] A. S. Mikhailov and K. Showalter, Phys. Rep. 425, 79 (2006).

[24] Pushpita Ghosh, Phys. Rev. E 84, 016222 (2011).

[25] M. Bertram and A. S. Mikhailov, Phys. Rev. E 63, 066102 (2001).

[26] V. Horvath, P. L. Gentili, V. K. Vanag, and I. R. Epstein, Angew. Chem. Int. Ed. 51, 6878 (2012).

[27] Y. Su, J. J. Wei, and J. P. Shi, J. Differ. Eq. 247, 1156 (2009).

[28] Y. Song, J. Xu, and J. Zhang, Chaos 21, 023111 (2011).

[29] C. Tian, Chaos 22, 013129 (2012).

[30] S. Grill, V. S. Zykov, and S. C. Muller, Phys. Rev. Lett. 75, 3368 (1995).

[31] U. Sommer, Algen, Quallen, Wasserfloh: Die Welt des Planktons (Springer-Verlag, Berlin, 1996).

[32] H. J. G. Baretta-Bekker, E. K. Duursma, and B. R. Kuipers, Encyclopedia of Marine Sciences (Springer-Verlag, Berlin, 1998).

[33] M. J. R. Fasham, Mar. Biol. Annu. Rev. 16, 43 (1978).

[34] S. V. Petrovskii and H. Malchow, Math. Comput. Modell. 29, 49 (1999).

[35] E. Beretta and Y. Kuang, J. Math. Anal. Appl. 204, 840 (1996).

[36] K. Gopalsamy, Bull. Math. Biol. 45, 295 (1983).

[37] A. Hastings, J. Math. Biol. 21, 35 (1984).

[38] R. M. May, Ecology 4, 315 (1973). 
[39] A. Martin and S. Ruan, J. Math. Biol. 43, 247 (2001).

[40] K. Gopalsamy, Stability and Oscillation in Delay Differential Equations of Population Dynamics (Kulwer Academic, Dordrecht, 1992).

[41] X. C. Zhang, G. Q. Sun, and Z. Jin, Phys. Rev. E 85, 021924 (2012).

[42] X. Lian, H. Wang, and W. Wang, J. Stat. Mech. (2013) P04006.

[43] See Supplemental Material at http://link.aps.org/supplemental/ 10.1103/PhysRevE.88.012713 for details of the dynamical process in Fig. 2.

[44] R. V. Ozmidov, Horizontal Turbulence and Turbulent Exchange in the Ocean (Nauka, Moscow, 1968).
[45] A. Okubo, Deep-Sea Res. 18, 789 (1971).

[46] A. Okubo, Diffusion and Ecological Problems: Mathematical Models (Springer-Verlag, Berlin, 1980).

[47] R. V. Ozmidov, Oceanology 38, 7 (1998).

[48] J. H. Steele, Spatial Pattern in Plankton Communities (Plenum Press, New York, 1978).

[49] M. Abbott, Phytoplankton Patchiness: Ecological Implications and Observation Methods in Patch Dynamics (Springer-Verlag, Berlin, 1993).

[50] I. R. Epstein and J. A. Pijman, An Introduction to Nonlinear Chemical Dynamics: Oscillations, Waves, Patterns, and Chaos (Oxford University Press, New York, 1998). 